\title{
Patients Initiated Timeline Marking of Events in Parkinson's Disease: Visualization of Time Correlation between Patients Marked Events and Acquired Data from Sensors
}

\author{
J. Artur Serrano ${ }^{1,2}$, Andrea Thoms ${ }^{3}$, Peter Weber $^{3}$, and SENSE-PARK Consortium \\ ${ }^{1}$ NST-Norwegian Centre for Telemedicine, University Hospital of North Norway, \\ Troms $\emptyset$, Norway \\ ${ }^{2}$ Department of Clinical Medicine, Faculty of Health Sciences, \\ The Arctic University of Norway \\ jarturserrano@gmail.com \\ ${ }^{3}$ HASOMED GmbH, Paul-Ecke-Straße 1, 39114 Magdeburg, Germany \\ \{andrea. thoms, peter. weber @hasomed. de
}

\begin{abstract}
The reality of a Parkinson's Disease patient involves coping with the condition 24 hours a day for the rest of her or his life. A continuous decay of physical and sometimes cognitive functions makes activities of daily life progressively more difficult to accomplish. Many keep a diary where they take note of feelings, relevant events related to the daily routines, reaction to the medication, etc. Such diaries may prove extremely useful for a better understanding of the disease progression, both by the patient and by the doctor. The SENSE-PARK project went a step forward: it combines the patient diary notes (self-reported) with information gathered from movement sensors (automatic measurement) and a visualization mechanism combining the two. A system has been designed, prototyped and tested. Parkinson's medical specialists, user experience experts, technologists and most important the patients themselves, were involved in this process.
\end{abstract}

Keywords: Parkinson's Disease (PD), People with Parkinson's (PwP's), patient diaries, marking of events, symptom, timeline, chronic disease management, self-management, second level prevention, activities of daily living (ADL), movement sensor, accelerometer, monitoring, degenerative disease, time correlation, User Centred Design (UCD), participatory design.

\section{$1 \quad$ Introduction}

Parkinson's is a degenerative disease where the impact of the condition is both difficult to measure and to manage, during the daily routines of the patient. To measure, because no methods or procedures are widely accepted, or recognised, by the scientific community, except from some tests done under medical supervision. To manage, because changes in condition and abilities to deal with daily tasks, or Activities of 
Daily Living (ADLs) are very different from patient to patient [1], and also because what you as patient can do and manage one day, can be extremely difficult the day after.

The limitations of a visit to the clinician do not allow for a complete view of the patient's evolving process: "Clinical visits provide only a brief snapshot of the condition that persons with Parkinson's disease (PD) present in that particular situation. However, PD is notorious for its fluctuations, which may occur both within and across days. Moreover, performance during the clinical visit does not always reflect how patients perform at home." [2]. The SENSE-PARK project [3] is trying to find ways to help People with Parkinson's (PwP's) to measure and manage their condition within their own lives.

The solution proposed in the SENSE-PARK project is a system consisting of hardware (sensors and mobile devices) and software. The sensors are detecting symptoms such as akinesia (absence, poverty, or loss of control of voluntary muscle movements), sway problems (related to balance), tremor and gait (problems with walking). The software helps identifying and showing to the user these symptoms based on the movement readings from the sensors. Nevertheless, there are still several problems to be resolved: the software may not always identify the symptom: either because it appears mixed with other movements; or because it is irregular and unclear; or still because it has a very small amplitude. All these factors are normally present and cause difficulties for automatic classification of the symptoms. Another issue is that the sensors do not detect what is the activity performed be the user at that time; for example, a tremor might occur during the activity of brushing the teeth. The sensor might detect the tremor, but the overlapping movement resulting from the teethbrushing activity is not recognised as it is not identified as being a teeth-brushing movement.

It is to solve these difficult problems that a user initiated activity marking system was introduced in the software. A PD patient, i.e. a user of the SENSE-PARK system, is able to "tell the system" what is happening (or, a posteriori, tell the system what happened at a certain time), for example, "now I am brushing my teeth", or "now I am vacuum cleaning", or "cycling" and so on. This is performed in a simple way: the user just has a function in the system to select the activity and then mark the "start activity". Once the activity is completed the user only has to choose the option "stop activity". This will then be shown in the system's user interface by a timeline with the sensor output and the corresponding activity markings.

As seen above, the system is used to mark what is known as the various ADLs. However, the system can be used, not only for the marking of ADLs, but also for marking the occurrence of symptoms, using the "start" and "stop" functionality, this time for the various symptoms such "Start tremor event" or "Start dyskinesia event". The marking of symptoms' events by the PD patient is mostly done a posteriori, when the user is visualizing what has just happened, looking in the system's user interface, or when is reviewing what happened during the day. 
The advantage of this system is to augment the capacity of the user in his / her ability to measure the condition he or her is living, both in relation to the ADLs and the symptoms. It also empowers the user to better manage the condition by being able to look back and see what factors can have an impact or in what degree they can affect the ability to perform ADLs and in what way the symptoms are correlated with such activities.

\section{Methods}

User Centred Design (UCD) was used from the start of the project. The users' needs analysis used a cross methods approach, with several focus groups and a web-based survey. Six focus groups sessions chaired by a person with Parkinson's and involving in total more than 15 PwP's as test users were organized in the United Kingdom, Spain and Sweden. A survey was conducted among people with Parkinson's disease. In 2011 the survey was distributed via the Web. A total number of 198 respondents answered the survey. Most of the respondents lived either in UK or USA, but there are a few from other countries. The results from the focus groups and the web-survey were cross analysed to produce a user requirements list.

During the UCD sessions, PwP's sometimes referred to their condition as a "journey". Facing the daily challenges proposed by the condition, observing the progression of the disease, learning and re-learning in a always novel process of how to deal with the effects of both the disease and the medication; this is what they call the "Parkinson's journey" [4]. The idea of a timeline seemed to correctly map the need of a support for the registration, visualization, referencing and analysis of events in context as they happen in the so-called "Parkinson's journey".

An Agile based method [5] was used for the prototype from design to implementation where several iterations of testing with PwP's were performed. Both technical feasibility and usability evaluation test were performed in each iteration.

The technical solutions of the SENSE-PARK project (sensors, App, PC-Software) were developed by the project partners HASOMED GmbH (www.hasomed.de) and HSG-IMIT (www.hsg-imit.de).

\section{$3 \quad$ Results}

A complete system has been created within the SENSE-PARK project. In Fig. 1 is shown the architecture of the system. It comprises a set of sensors to be fitted to the user, a mobile App to be installed in the user smart phone and a PC to upload the data regarding the user activities and the data collected by the sensors. This data is then displayed in a diagram, where the sensor data (automatically acquired) is time correlated with the activities (user marked events). 


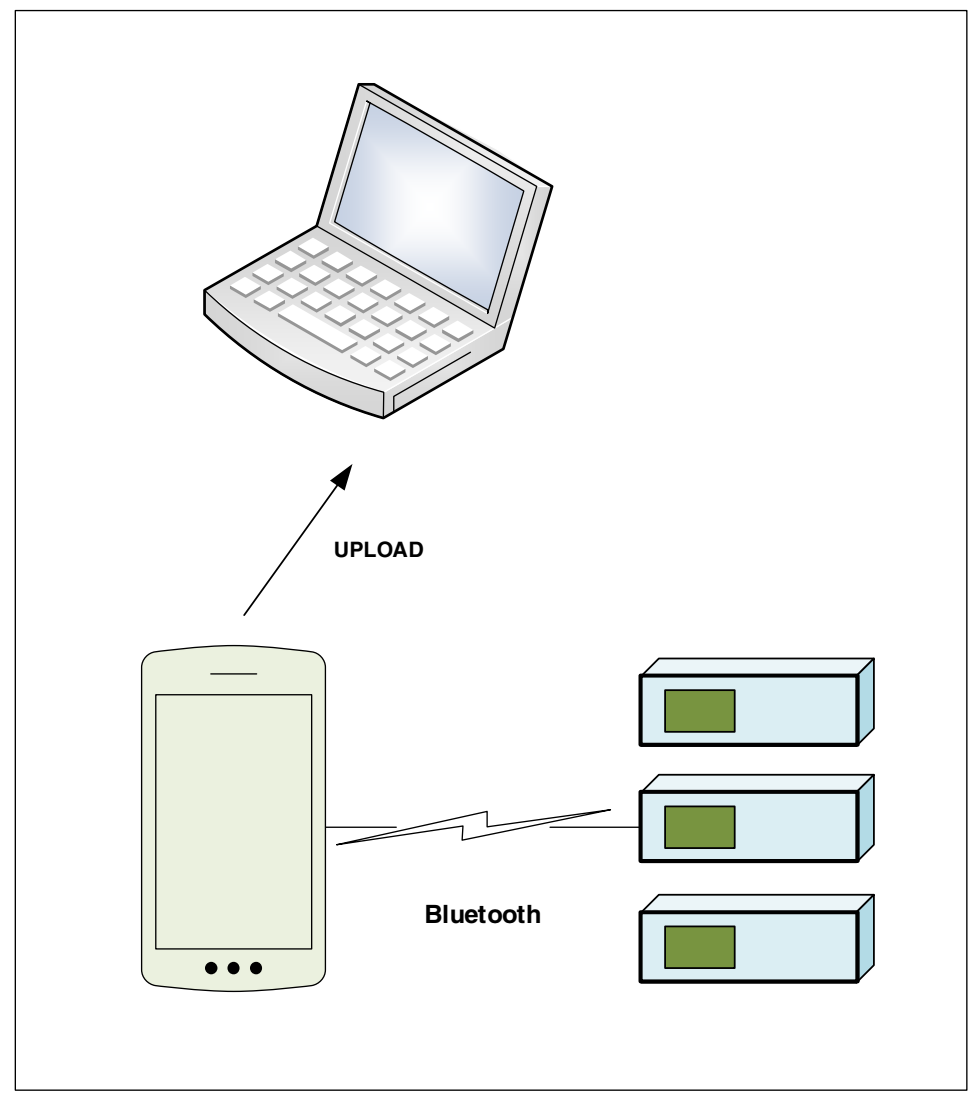

Fig. 1. Architecture

\subsection{The SENSE-PARK App}

An app was developed following the methodology presented above and based on the results of the User Needs Analysis with the strong participation of PWP's. The app is called PDApp ${ }^{1}$ (PD from Parkinson's Disease) and is executed on an Android-based smart phone.

The system comprises the app itself and a set of sensors [6] to be worn by the patient. A sensor should be used on one or both wrists, the same on the feet and one on the waist. The communication between the PDApp and the sensors is done using Bluetooth.

\footnotetext{
${ }^{1}$ The programming of the app was performed by HASOMED GmbH within the scope of the SENSE-PARK project. The sensors described in this article are also a HASOMED product and have been developed prior to the project.
} 

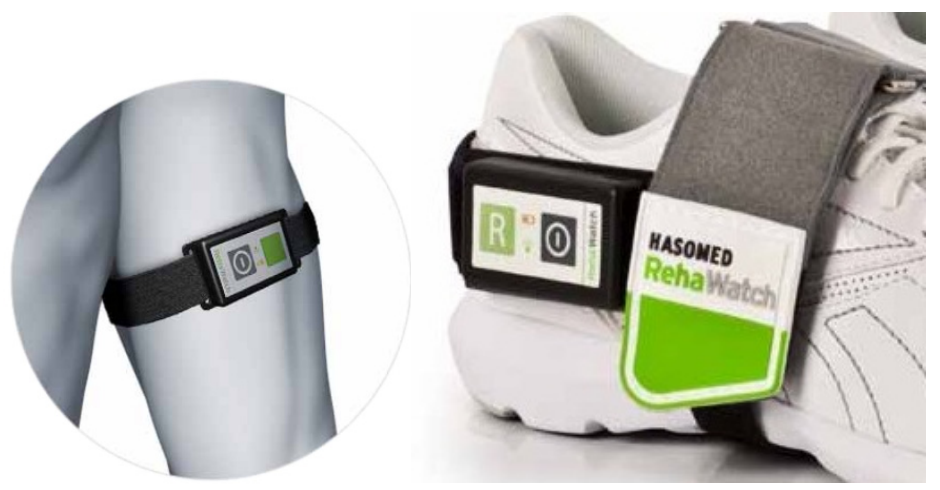

Fig. 2. Method of fitting the sensors using a strap

The PDApp allows for the registration of user reported events and sensor data. The user reported events and the automatic data collection performed by the sensors can be time correlated as both types of data are time-stamped and the internal clock of the sensors is synchronized with the smart phone clock. The time adjustment in the sensors is done at PC upload time. As this task is performed daily, the eventual time fluctuation within the sensors is negligible.

\subsection{Monitoring Activities}

Different activities can be created by the user, such as "Walking", "Drinking" or "Cooking". They can be subsequently edited or deleted (Fig. 3).

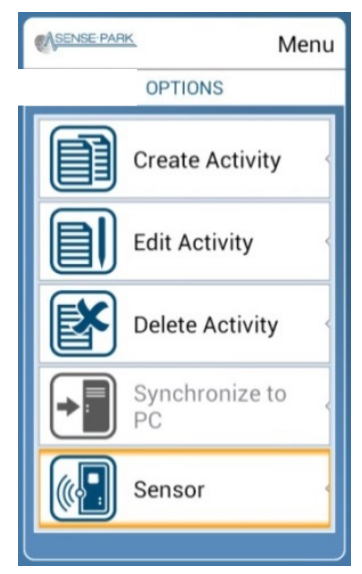

Fig. 3. Activities' set-up

An activity can be given a name chosen by the user. The user also defines an instruction (a text explaining what should be done to perform this activity) and timing options for start and stop of the activity (Fig. 4). 


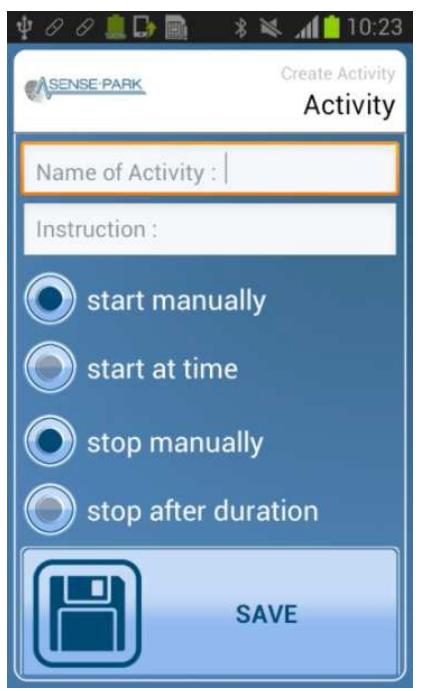

Fig. 4. Activity creation screen

When the system is in use, a given activity can be displayed. The activity is displayed with the instruction of what to do. If the activity was set-up with manual start the user can initiate it by pressing the START button. If the activity has been set-up with manual stop, then START button changes into the STOP button, so that the activity can be stopped by the user (Fig. 5).
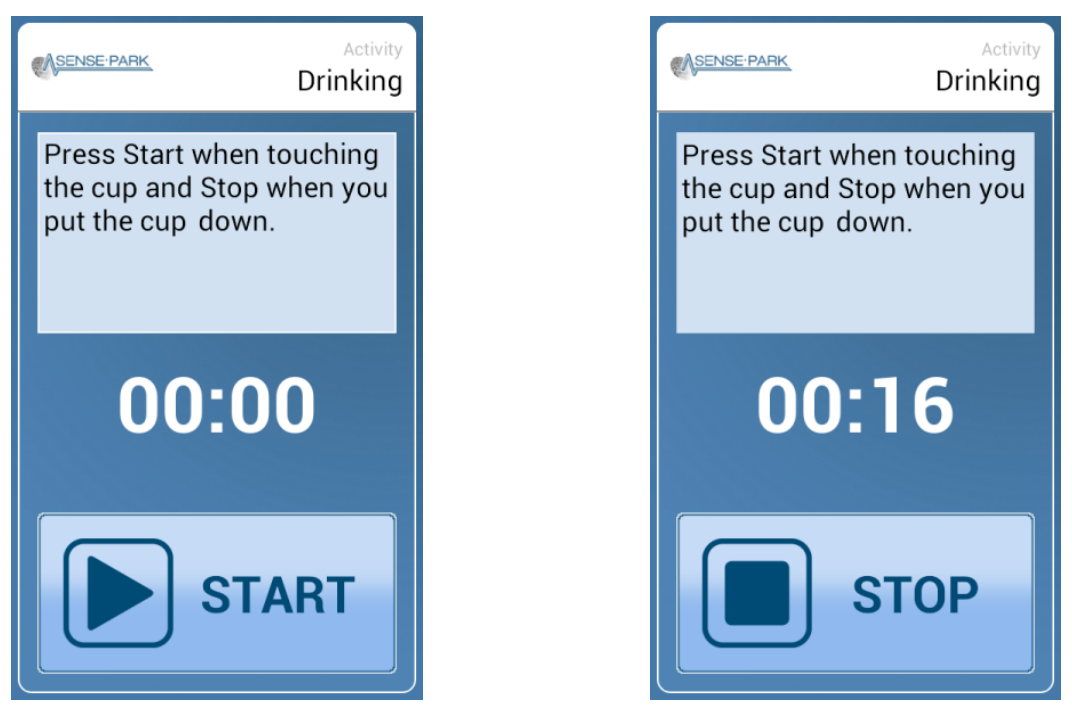

Fig. 5. App displaying the instructions for the activity "Drinking" (left) and App during the execution of this activity (right) 


\subsection{Visualization of the Activities and the Time Correlation with the Acquired Data from Sensors}

At the end of the day (for day sensors), and in the morning (for night sensors), the user connects the Android device to the PC via a USB cable. This way, all sensor data and activity information is upload into the PC. The imported data can be visualized on the PC. In Fig. 6 an example is shown for acquired data regarding akinesia, which is the absence, poverty, or loss of control of voluntary muscle movements. The figure also show the time correlated data of the "walking" activity.

It can be seen that after the start of the walking activity there was a period of full movement (defined at a value of 80 , as this is the maximum value measured by the sensors), which was followed by a short period of complete akinesia (no movement) and then full movement once again. To notice that a marking of a mood was also expressed, which may be given by the user to the system in a similar way as it was described for an activity. A mood is however an indication of a feeling in a particular instant of time.

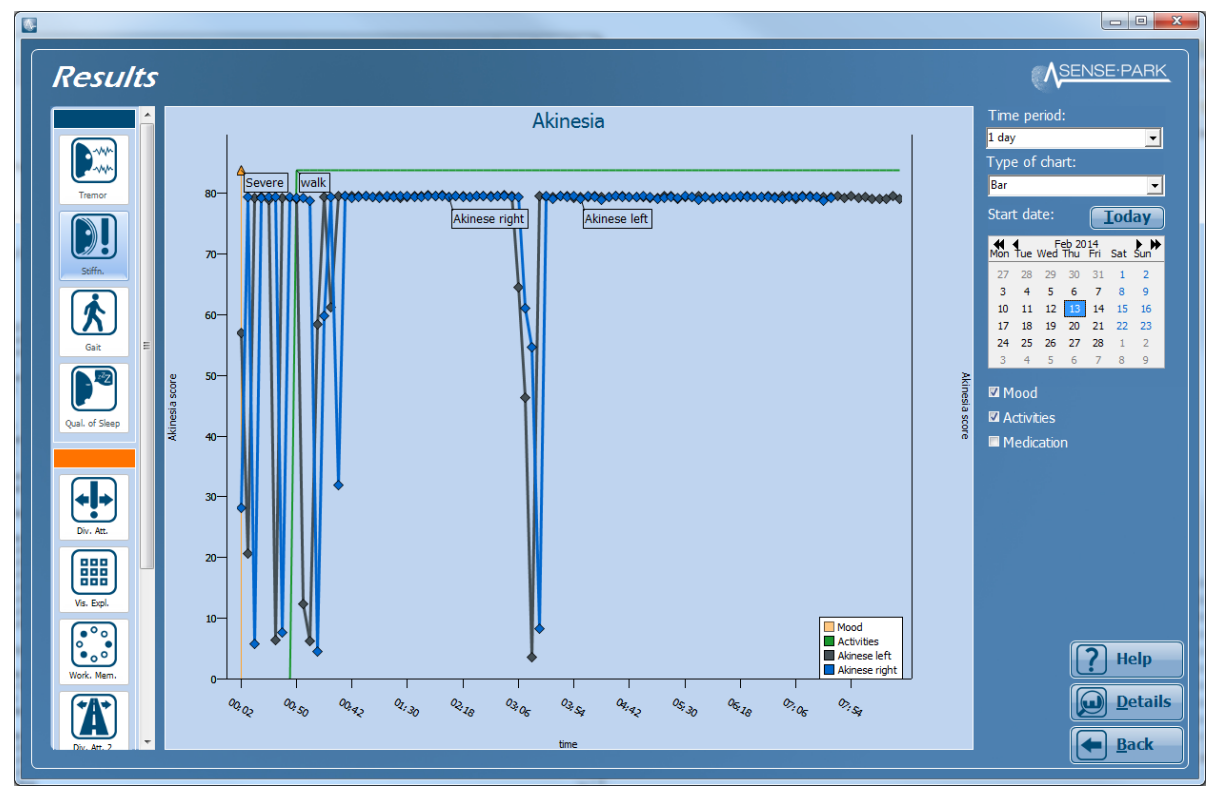

Fig. 6. The presented akinesia score has a range from 0 , or no movement (complete akinesia) to 80 , or full movement (no detectable akinesia)

\section{Discussion}

"Being" and "becoming" are two requirements that PwP's express when confronted with the disease. The attitude of the patient to the present - "being" - and the future "becoming" - defines how successful the "parkinson's journey" may turn out. The 
present of being a patient, and the future in part defined by the progression of the condition, create the experience of "losses of physical and mental functioning and independence, self-identity and future" [7]. The PDApp allows the PD patient to record a timeline of events of several natures, which can consist of:

- psychological: moods;

- ADLs: routine activities;

- medication: what and how it is used;

- output from other systems, for example applied games;

- social context: where I was, with whom and for what purpose.

All these provide information about the patient and the disease. Not in a single snapshot, but on a continuous manner, reflecting both the internal conditions of the person such as mood, physical state, tiredness, and the external factors such as taken medication, social context and activities being done.

The system developed within the SENSE-PARK project seems to be able to provide a support to acquire, register and visualize such information. Moreover, we argue that the active engagement of the patient in recording his or her data and later visualizing it, may provide additional motivation to actively manage the condition: "Reversing the sedentary lifestyle of elderly subjects and patients with neurodegenerative disorders could have beneficial effects on gait and balance, achieve generic health benefits and increase survival [8] (...) motivating a sedentary person to become more active even in the long term is a challenging task. (...) strategies to increase motivation and self-engagement create new opportunities to achieve a more active lifestyle and treatment compliance" [9].

For the clinician, the data can later be exported into a spreadsheet, which, once anonymized, can be used in clinical research.

The usefulness of this approach is currently being investigated within the project. Once the system is ready for trial, both clinicians and patients will be searching for the necessary evidence that proves such usefulness.

\section{Further Work}

Events can also be triggered by medical devices or other software systems. As an example, an automated medication system can be connected to the PDApp. In this case a medication reminder can be created to behave as any other activity. Once the automated medication system generates the alarm (Fig. 7), an activity can be set-up to start automatically. A screen will appear where you are able to choose a state for the medication taking (Fig. 8). A prototype of this system has already been produced. Different visualization techniques are currently under development for the display of the data. 


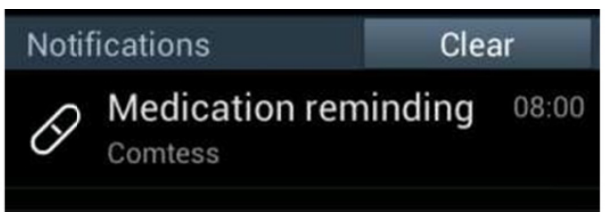

Fig. 7. Medication reminder

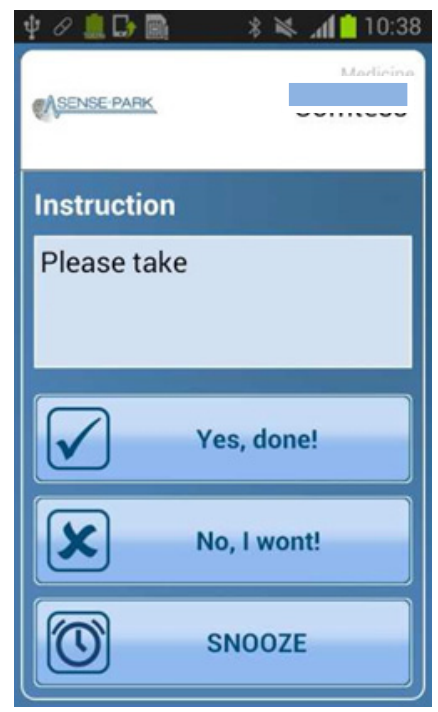

Fig. 8. After a medication reminding alarm, the user is presented with several options

When all activities are time correlated, for example medication taken, exercise and with episodes of akinesia or tremor, then it is possible to investigate the influence of one upon another. How does the combination of exercise and a particular medication influence the occurrence of akinesia? For example.

The statistical analysis of time correlated data may provide important information to the further understanding of the Parkinson's disease.

Moods, activities, external medical devices and software, sensor data for different domains, results from cognitive assessment games, all these could be introduced in the PDApp and visualized in the user interface.

A clinical study to test the feasibility of continuous quantitative gait, balance, tremor, hypoykinesia, dyskinesia, sleep and cognition assessment of PD patients in a home environment over a time period of 16 weeks is planned to start in the summer of this year (2014). The multi-centre study will be performed in 3 countries and will comprise 24 participants.

Results from this study will provide guidance and further requirements for the development of the PDApp. 
Acknowledgements. The present research is part of the EU project SENSE-PARK, funded under the Seventh Framework Programme, Cooperation - ICT, Grant Agreement no. 288557.

\section{References}

1. Hobson, J.P., Edwards, N.I., Meara, R.J.: The Parkinson's Disease Activities of Daily Living Scale: a new simple and brief subjective measure of disability in Parkinson's disease. Clin. Rehabil. 15, 3241-3246 (2001), doi:10.1191/026921501666767060

2. Maetzler, W., Domingos, J., Srulijes, K., Ferreira, J.J., Bloem, B.R.: Quantitative wearable sensors for objective assessment of PD. Mov. Disord. 28 (2013), doi:10.1002/mds.25628

3. SENSE-PARK website, http: / / www . sense-park . eu

4. Isaacs, T.: Shake Well Before Use. Cure Parkinsons Press, London (December 6, 2007) ISBN-10: 0955773008

5. Greer, D., Hamon, Y.: Agile Software Development. Softw: Pract. Exper. 41(9), 943-944 (2011), doi:10.1002/spe.1100

6. RehaWatch, http: //www. hasomed. de/en/products/rehawatch-gait-analysis.html

7. Charlton, G.S., Barrow, C.J.: Coping and self-help group membership in Parkinson's disease: an exploratory qualitative study. Journal of Health \& Social Care in the Community 10(6), 472-478 (2002), doi:10.1046/j.1365-2524.2002.00385.x

8. Lee, I.M., Shiroma, E.J., Lobelo, F., Puska, P.: Effect of physical inactivity on major noncommunicable diseases worldwide: an analysis of burden of disease and life expectancy. Lancet. 380, 219-229 (2012), doi:10.1016/S0140-6736(12)61031-9

9. Maetzler, W., Nieuwhof, F., Hasmann, S.E., Bloem, B.R.: Emerging therapies for gait disability and balance impairment: promises and pitfalls. Mov. Disord. 28(11), 1576-1586 (2013), doi:10.1002/mds.25682 